This item was submitted to Loughborough's Research Repository by the author.

Items in Figshare are protected by copyright, with all rights reserved, unless otherwise indicated.

\title{
On importance of surface forces in a microfluidic fluidized bed
}

PLEASE CITE THE PUBLISHED VERSION

http://dx.doi.org/10.1016/j.ces.2014.12.023

\section{PUBLISHER}

(c) Elsevier Ltd

\section{VERSION}

VoR (Version of Record)

\section{PUBLISHER STATEMENT}

This work is made available according to the conditions of the Creative Commons Attribution-NonCommercialNoDerivatives 4.0 International (CC BY-NC-ND 4.0) licence. Full details of this licence are available at: https://creativecommons.org/licenses/by-nc-nd/4.0/

\section{LICENCE}

CC BY-NC-ND 4.0

\section{REPOSITORY RECORD}

Zivkovic, Vladimir, and Mark J. Biggs. 2019. "On Importance of Surface Forces in a Microfluidic Fluidized Bed”. figshare. https://hdl.handle.net/2134/18657. 


\title{
On importance of surface forces in a microfluidic fluidized bed
}

\author{
V. Zivkovic ${ }^{*, 1,2}$ and M.J. Biggs ${ }^{2,3}$ \\ ${ }^{1}$ School of Chemical Engineering and Advanced Materials, Newcastle University, Merz \\ Court, Newcastle-upon-Tyne, NE1 7RU,UK \\ ${ }^{2}$ School of Chemical Engineering, The University of Adelaide, Adelaide, SA 5005, \\ Australia \\ ${ }^{3}$ School of Science, Loughborough University, Loughborough, LE11 3TU, UK \\ *Email: vladimir.zivkovic@ncl.ac.uk
}

\begin{abstract}
Fluidized beds potentially offer a means of significantly enhancing mixing, heat and mass transfer under the low Reynolds number flow conditions that prevail in microfluidic devices. However, as surface forces at the microscale can be significant relative to hydrodynamics forces, fluidization within a microfluidic channel can be potentially hindered or even prevented through particle adhesion to the channel walls. We have used the acid-base theory of van Oss, Chaudhury and Good to predict the propensity for adhesion of particles on microfluidic fluidized bed walls for various practically important wall material/particle/fluid combinations. Comparison of the results from this approach with experimental observations indicates it provides a robust means of predicting the adhesion propensity. It is also demonstrated how results from the model can be used to estimate for a system of interest the particle size range in which the particle-wall surface forces transition from being dominate to being insignificant.
\end{abstract}

Keywords: Fluidization; Interfacial tension; Multiphase flow; Microfluidics; Microfluidized bed; Process intensification. 


\section{INTRODUCTION}

Microfluidics (Squires and Quake, 2005; Stone et al., 2004; Whitesides, 2006) is the science and technology of processing of small volumes of fluids in conduits having dimensions of the order of tens to hundreds of micrometres. This research area holds promise in disparate fields ranging from automation of chemical analysis (Dittrich et al.,

2006; Manz et al., 1990; West et al., 2008) to medical diagnostics (Abgrall and Gué, 2007; Haeberle and Zengerle, 2007; Melin and Quake, 2007) through to process intensification (Charpentier, 2005; Dudukovic, 2009; Haswell, 2006; Jensen, 2001). This promise is frustrated, however, because the heat and mass transport central to these and other applications is dominated by the molecular diffusion that comes with the inevitable laminar flow found in micron-sized conduits. Fluidized beds have long been used at the macro-scale to enhance mixing and, thereby, heat and mass transport. They have, however, not been exploited at all in the microfluidics context. Recent modelling (Derksen, 2008, 2009) and experimental work (Doroodchi et al., 2012; Potic et al., 2005; Zivkovic et al., 2013a; Zivkovic et al., 2013b) has demonstrated that microfluidic fluidized beds (termed henceforth 'microfluidized beds') are feasible, offering the potential to not only overcome diffusion-limited heat and mass transport in simple micron-sized channels, but also provide higher sensitivity and multi-modal detection in the diagnostic context by virtue of the large surface area per unit volume that comes from use of micro-particles (Derveaux et al., 2008; Lim and Zhang, 2007).

The main difference between micro- and macro-scale flows is the importance of surface forces relative to volumetric forces such as gravity. Some have used the cross-sectional size of the conduit as the basis for differentiating between the two regimes, with $1 \mathrm{~mm}$ being the widely asserted boundary (Gunther and Jensen, 2006; Hartman and Jensen, 
2009). The situation is, however, more nuanced than this. In our recent work with a sub-500 $\mu \mathrm{m}$ microfluidized bed (Zivkovic et al., 2013a), for example, we have noted both particle adhesion onto the bed walls and subsequent de-fluidization and smooth, well behaved fluidization depending on the fluidizing liquid used. This suggests a more robust means is required to determine when surface forces are likely to be of concern in microfluidized beds.

Here we show that the acid-base theory developed by van Oss, Chaudhury and Good (van Oss, 2008) can successfully predict particle adhesion to the walls of microfluidized beds and its absence as a function of the fluidizing liquid and solid materials involved. The structure of this paper is as follows. We will first outline the experimental details, including the apparatus, the particulate and liquid materials, and the experimental procedures used. Secondly, a brief theoretical background for adhesion between a solid wall and particles immersed in a liquid is given. This is followed by a presentation and discussion of the results obtained using this theory, including comparison with experiment, and, finally, conclusions.

\section{EXPERIMENTAL DETAILS}

\section{Microfluidic bed}

The microfluidic beds used in the work reported here are shown in Fig. 1. The microchannels from which the beds were formed were fabricated in a polydimethylsiloxane (PDMS) chip by standard soft lithography techniques (Whitesides et al., 2001); this approach was used because of its relative simplicity, low cost and rapidity of manufacture (Becker and Gärtner, 2008). The cross-sectional dimensions of the microchannels were $400 \times 175 \mu \mathrm{m}$, whilst the lengths were typically around 20 $\mathrm{mm}$. The precision of the microfluidics manufacturing process used here is around $1 \mu \mathrm{m}$ 
as confirmed by casting the channels and cutting for examination by an optical microscope. The design of the distributor evolved throughout our study, although it remained constrained by the requirement that it should be relatively easy to fabricate using standard soft lithography techniques. The first design was the simple dam distributor (380 $\mu \mathrm{m}$ wide with $10 \mu \mathrm{m}$ gaps on both side) shown in Fig. 1(a), which was inspired by the non-conventional distributor of Potic et al. (2005). This design was particularly attractive due to the ease of manufacture, but experience as well as computational fluid dynamics (Zivkovic et al., 2010; Zivkovic et al., 2013c) showed that fluid flow was not as uniform as would be liked. This simple design was, therefore, replaced by the circular pillar distributor shown in Fig. 1(b), which computational fluid dynamics indicated would lead to more uniform fluid flow (Zivkovic et al., 2010). In this design, the distributor consisted of eight circular pillars of $40 \mu \mathrm{m}$ diameter separated by $9 \mu \mathrm{m}$ gaps. Unfortunately, this design was mechanically weak and, hence, we ultimately adopted the mechanically more robust distributor design shown in Fig. 1(c), which is a series of five $70 \mu \mathrm{m}$ wide and $200 \mu \mathrm{m}$ long rectangular pillars separated again by 8-9 $\mu$ m gaps. (Zivkovic et al., 2013a). One issue with this design is that during the bonding process of the microchannels, there is some bending of the pillars due to PDMS flexibility and their high-aspect ratio. This leads to the individual gaps being somewhat non-uniform in size as can be seen in the Fig. 1(c). Despite this, visual observation during the fluidization experiments indicated that none of the gaps were blocked and that the fluid flow was relatively uniform. 


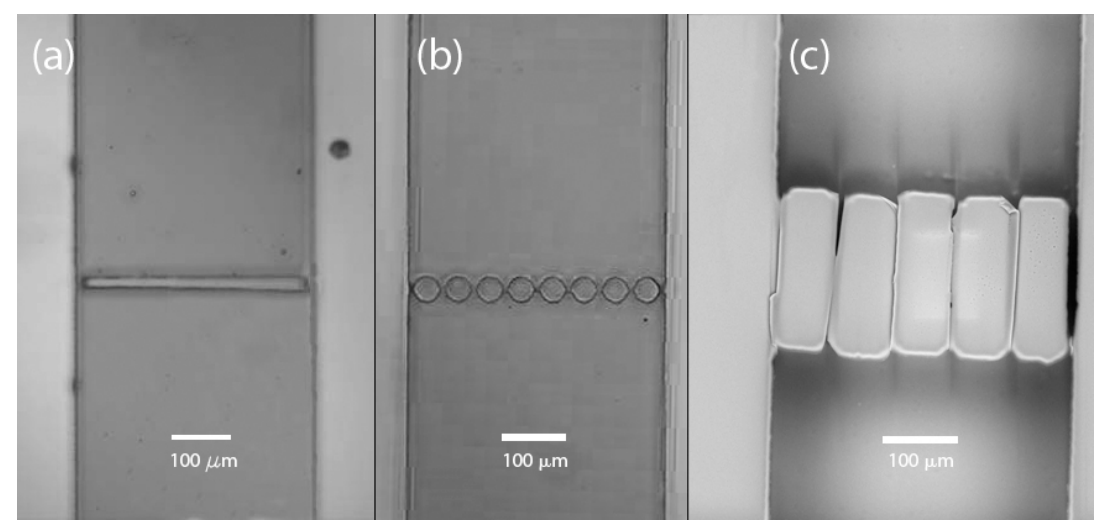

Fig. 1: Optical micrograph showing the three distributor designs used in the work reported here: (a) dam distributor; (b) series of circular pillars; and (c) series of rectangular pillars.

The schematic of the experimental setup is shown in Fig. 2. The chip containing the microfluidized bed was mounted vertically in a custom made holder on a heightadjustable rotary stage (M488, Newport Corporation, US) for easy manipulation. A trinocular stereomicroscope with holder and fibre optic illuminator (SMZ-168-TH, Motic, China) connected to a digital camera (KY-F550E, JVC, Japan) was used for recording movies. The digital movies were stored on a PC for offline analysis. The fluidizing liquid was pumped by the PHD ULTRA syringe pump (Harvard Apparatus, US) at desired flow rates. Experiments were performed at room temperature of $25 \pm$ $1^{\circ} \mathrm{C}$.

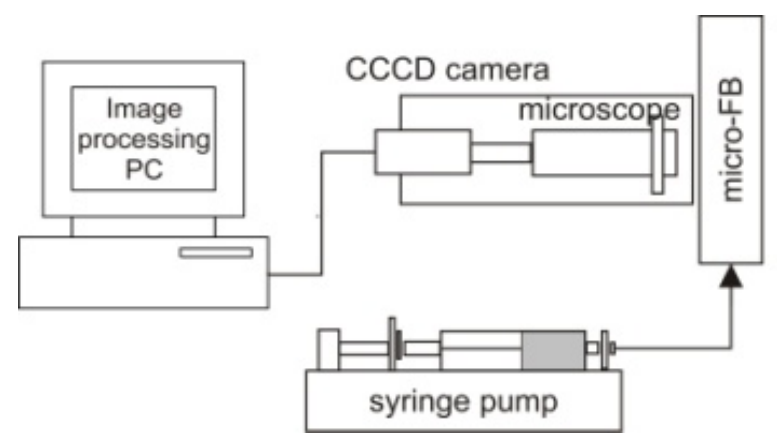

Fig. 2: Schematic of experimental setup for the top-view flow visualization. 


\section{Particulate and Liquid Materials}

We considered two different groups of as-supplied particles: (1) four different sized soda lime glass microspheres of density $\rho_{p}=2500 \mathrm{~kg} / \mathrm{m}^{3}$ and average diameter $d_{p}=$ $26.5,30.5,34.5$ and $38.5 \mu \mathrm{m}$ with a standard deviation of $1.5 \mu \mathrm{m}$; and (2) four different sized poly(methyl methacrylate) (PMMA) particles (Cospheric LLC, US) of density $\rho_{p}=1120 \mathrm{~kg} / \mathrm{m}^{3}$ and average diameter $d_{p}=23.5,29.5,35.0$ and $41.5 \mu \mathrm{m}$ with a standard deviation of $3.5 \mu \mathrm{m}$. Fluidization experiments were undertaken using three different liquids: deionized water, deionized water containing 0.15 g/l Tween 80 surfactant ( $\geq 99.5 \%$, Sigma Aldrich, St. Louis, Missouri, US), and anhydrous ethanol ( $\geq$ 99.5\%, Sigma Aldrich, St. Louis, Missouri, US). The deionized water was obtained from an ELGA PURELAB (VWS Ltd., UK) classic water purification system.

\section{Theoretical Background}

A particle will prefer to adhere to a solid surface if its free energy decreases as it moves from the bulk phase to the surface (i.e. $G$ (surface) $-G$ (bulk) $<0$ ) . In the acid-base approach developed by van Oss, Chaudhury and Good (van Oss, 2008), the free energy of interaction between two different solid surfaces immersed in a liquid may be expressed as

$$
\begin{gathered}
\Delta G_{1 w 2}=\left(\sqrt{\gamma_{1}^{L W}}-\sqrt{\gamma_{2}^{L W}}\right)^{2}-\left(\sqrt{\gamma_{1}^{L W}}-\sqrt{\gamma_{w}^{L W}}\right)^{2}-\left(\sqrt{\gamma_{2}^{L W}}-\sqrt{\gamma_{w}^{L W}}\right)^{2}+ \\
2\left[\begin{array}{c}
\sqrt{\gamma_{w}^{+}}\left(\sqrt{\gamma_{1}^{-}}+\sqrt{\gamma_{2}^{-}}-\sqrt{\gamma_{w}^{-}}\right)+\sqrt{\gamma_{w}^{-}}\left(\sqrt{\gamma_{1}^{+}}+\sqrt{\gamma_{2}^{+}}-\sqrt{\gamma_{w}^{+}}\right) \\
-\sqrt{\gamma_{1}^{+} \gamma_{2}^{-}}-\sqrt{\gamma_{1}^{-} \gamma_{2}^{+}}
\end{array}\right]
\end{gathered}
$$

where the subscripts 1,2 and $w$ relate to the two solid surfaces and liquid respectively, and the surface energy, $\gamma$, of the liquid and solid surfaces is expressed as a sum of an apolar (Lifshitz-Van der Waals) component, $\gamma^{L W}$, and a polar (Lewis acid-base) 
component, $\gamma^{A B}$, that is in-turn expressible as twice the geometric mean of a Lewis acid (electron-donating) component, $\gamma^{-}$, and a Lewis base (electron-accepting) component $\gamma^{+}$, giving

$$
\gamma=\gamma^{L W}+\gamma^{A B}=\gamma^{L W}+2 \sqrt{\gamma^{+} \gamma^{-}}
$$

The absolute values of $\gamma^{-}$and $\gamma^{+}$are at present unknown for any material. Thus, in order to be able to express and measure $\gamma^{-}$and $\gamma^{+}$for various liquids and solid surfaces, it is assumed that the ratio $\gamma^{-} / \gamma^{+}$is unity for water, at $20^{\circ} \mathrm{C}\left(\right.$ i.e. $\left.\gamma^{-}=\gamma^{+}=25.5 \mathrm{~mJ} / \mathrm{m}^{2}\right)(\mathrm{van}$ Oss, 2008).

Of course, even if a particle has a tendency to adhere to the wall of a micro-fluidized bed (i.e. $\Delta G_{1 w 2}<0$ ), whether it does or not depends on how the adhesion force compares to the hydrodynamic force it experiences. The Derjaguin approximation can be used to determine the former given the free energy obtained from the acid-base approach (van Oss, 2008). Assuming the particles and the bed wall can be approximated by spheres and a flat plate respectively, this approximation leads to (van Oss, 2008)

$F_{a d h}=\pi \Delta G_{1 w 2} d_{p}$

In the case of the hydrodynamic force experienced by the particle, its unambiguous evaluation is challenging because of the existence of a number of disparate expressions for the force (see, for example, Di Felice (1995)) along with their omission of the wall effects that are bound to be important here. An alternative way of approximating the hydrodynamic force experienced by the particle is through equating it to the drag force, $F_{d}$, that can in turn be equated through a simple force balance to the difference between the particle weight, $W$, and buoyancy force, $F_{b}$

$F_{d}=W-F_{b}$ 
Whilst there is also some debate around the buoyancy force (Di Felice, 1995; Epstein, 2003), it is more straightforward to evaluate. Here we use the static buoyancy of Clift (1987)

$F_{b}=V_{p} \rho_{f} g$

where $\rho_{f}$ is the fluid density, $g$ the acceleration due to gravity, and $V_{p}$ the particle volume. This gives a more conservative estimate of the drag force than that of Gibilaro (Di Felice, 1995; Epstein, 2003), which would yield a smaller value by a factor of the voidage. Using Eq. 5 in Eq. 4 yields

$F_{d}=\left(\frac{\pi d_{p}^{3}}{6}\right)\left(\rho_{p}-\rho_{f}\right) g$

\section{RESULTS AND DISCUSSION}

\section{Free Energy Predictions for Experimental Systems}

The experimental determination of surface tension is notoriously fraught because of its sensitivity to contaminants and environmental conditions. This means the literature contains different values for nominally the same quantity as shown in Table 1 for the two liquids (water and ethanol) and three solids (PDMS, PMMA and glass) considered in our experimental study. The free energies of interaction, $\Delta G_{1 w 2}$, for the various wall material/particle/ liquid triplets were evaluated using all combinations of these values in Eq. 1. The free energies obtained from the combinations for a triplet were then used to obtain the averages and standard deviations shown in Table 2 along with the associated minima and maxima. 
Table1: Surface tension components (in $\mathrm{mJ} / \mathrm{m}^{2}$ ) for liquids and solid used in this study taken from various literature sources.

\begin{tabular}{ccccccl}
\hline Material & $\gamma$ & $\gamma^{L W}$ & $\gamma^{A B}$ & $\gamma^{+}$ & $\gamma^{-}$ & Reference \\
\hline Water & 72.8 & 21.8 & 51.0 & 25.5 & 25.5 & van Oss (2008) \\
\hline Ethanol & 22.4 & 20.1 & 2.3 & 0.1 & 13 & van Oss (1989) \\
& 21.4 & 18.8 & 2.6 & 0.02 & 68 & van Oss (2008) \\
& 23.2 & 21.4 & 1.8 & 0.09 & 9 & Zdziennicka (2010) \\
\hline Glass & 59.8 & 42.0 & 17.8 & 1.97 & 40.22 & Freitas \& Sharma (1999) \\
& 64.37 & 42.3 & 22.07 & 2.9 & 42.0 & Clint \& Wicks (2001) \\
& 51.7 & 33.7 & 18.0 & 1.3 & 62.2 & van Oss (2008) \\
\hline PMMA & 44.65 & 42 & 2.65 & 0.55 & 3.2 & Della Volpe \& Siboni (2000) \\
& 44.58 & 41.2 & 3.38 & 0.38 & 7.5 & Clint \& Wicks (2001) \\
& 40.6 & 40.6 & 0 & 0 & 12 & van Oss (2008) \\
& 39.21 & 36.68 & 2.53 & 0.16 & 10.02 & Zdziennicka (2010) \\
\hline PDMS & 25.46 & 18.4 & 7.06 & 4.37 & 2.86 & Chae et al. (2009) \\
& 13.45 & 10.8 & 2.65 & 0.4 & 4.4 & Hu et al. (2010) \\
& 18.6 & 18 & 0.60 & 0.13 & 0.7 & Martinelli et al. (2011) \\
& 22.3 & 21.8 & 0.58 & 0.39 & 0.22 & Martinelli et al. (2011) \\
\hline
\end{tabular}

Table 2: Free energy of interaction (in $\mathrm{mJ} / \mathrm{m}^{2}$ ) between glass or PMMA particles and the sidewalls of the PDMS microfluidized bed in ethanol and water. The mean, $\left\langle\Delta G_{1 w 2}\right\rangle$, standard deviation, $\sigma(\Delta G)$, minimum, $(\Delta G)_{\min }$, and maximum, $(\Delta G)_{\max }$, values are obtained by using different sets of the surface tension parameters drawn from Table 1.

\begin{tabular}{cccccccc}
\hline Surf. 1 & Surf. 2 & Liquid & $\left\langle\Delta \boldsymbol{G}_{\mathbf{1} \boldsymbol{w} \mathbf{2}}\right\rangle$ & $\boldsymbol{\sigma}(\boldsymbol{\Delta G})$ & $(\Delta \boldsymbol{G})_{\min }$ & $(\Delta \boldsymbol{G})_{\max }$ & Interaction \\
\hline Glass & PDMS & Water & -10.42 & 7.64 & -21.97 & 5.12 & Attractive \\
PMMA & PDMS & Water & -51.55 & 9.92 & -68.33 & -39.11 & Attractive \\
Glass & PDMS & Ethanol & 7.90 & 10.85 & -14.50 & 27.55 & Repulsive \\
PMMA & PDMS & Ethanol & 6.32 & 7.58 & -2.60 & 33.24 & Repulsive \\
\hline
\end{tabular}

All calculations but one predict glass microparticles will be attracted to a PDMS wall when immersed in water; only when the PDMS parameters of Hu et al. (2010) are used with the glass parameters of van Oss (2008) do we get a positive free energy ( $\Delta G_{1 \mathrm{w} 2}=$ $5.12 \mathrm{~mJ} / \mathrm{m}^{2}$ ) indicating repulsion between the two materials in the presence of water. However, as Table 2 shows, the average of the free energies for this combination is $-10.42 \mathrm{~mJ} / \mathrm{m}^{2}$ with a standard deviation of $7.64 \mathrm{~mJ} / \mathrm{m}^{2}$, which suggests that glass 
microparticles almost certainly have a propensity to adhere to a PDMS surface in the presence of water.

In the second case involving PMMA particles in the presence of water, all the evaluated free energies are substantially less than zero, perhaps not unsurprisingly given the hydrophobicity of both PMMA and PDMS. With an average free energy of interaction of $-51.55 \mathrm{~mJ} / \mathrm{m}^{2}$ and standard deviation of $9.92 \mathrm{~mJ} / \mathrm{m}^{2}$ (Table 2), the theory suggests a significant propensity for adhesion of PMMA particles to PDMS surfaces.

In the third case of glass particles immersed in ethanol, the majority of the parameter combinations yield positive free energies except those involving the PDMS parameters of Chae et al. (2009) and either the van Oss (1989) or Zdziennicka (2010) ethanol parameters (6 out of 36). Although the average free energy for the latter six combinations suggest some propensity for adhesion (mean value of $-8.37 \mathrm{~mJ} / \mathrm{m}^{2}$ with standard deviation of $4.00 \mathrm{~mJ} / \mathrm{m}^{2}$ ), the average of all 36 combinations is $7.89 \mathrm{~mJ} / \mathrm{m}^{2}$ with a standard deviation of $10.85 \mathrm{~mJ} / \mathrm{m}^{2}$, suggesting little propensity for glass particle adhesion to PDMS surfaces in the presence of ethanol.

In the last case of PMMA particles in ethanol, the mean and standard deviation of the free energies obtained from all 48 parameter combinations are $6.32 \mathrm{~mJ} / \mathrm{m}^{2}$ and 7.58 $\mathrm{mJ} / \mathrm{m}^{2}$ respectively, whilst the counterparts for the five of these combinations that are attractive are $-1.33 \mathrm{~mJ} / \mathrm{m}^{2}$ and $1.00 \mathrm{~mJ} / \mathrm{m}^{2}$ respectively. These results strongly suggest that the adhesion propensity for PMMA particle adhesion to PDMS surfaces is negligible in the presence of ethanol. 


\section{Comparison of Adhesion/Drag Force Ratio Predictions with Experimental Observations}

Although the free energy allows identification of the propensity for particle adhesion to a surface, the ratio of the adhesion and drag forces experienced by a particle in the micro-fluidised bed is required to understand if adhesion to the bed wall will in fact occur. Table 3 shows for the various wall material/particle/liquid triplets the estimated adhesion force derived using the Derjaguin approximation, Eq. 3, the drag force evaluated from Eq. 6, and their ratio. In both the cases of glass and PMMA particles fluidized by water in a PDMS microfluidized bed, adhesion forces are some 3 to 5 orders of magnitude larger than the estimated drag forces, indicating particle adhesion to the bed walls is highly likely in these cases. This was indeed reflected in our efforts to fluidize the glass particles in the microfluidic bed using deionized water (Zivkovic et al., 2013a), which proved impossible due to the particles adhering to the walls of the microfluidized bed as shown in Fig. 3. Furthermore, in our latest experiments we also observed the same behaviour (not shown here) when we sought to fluidize PMMA microparticles with water in a microfluidic bed having a rectangular pillar distributor.

Table 3: Estimates of adhesion force, $F_{a d h}$, buoyant weight of particles, $F_{b w}$, and ratio of these forces for glass and PMMA particles in a PDMS microfluidized bed using ethanol and water as the fluidizing liquid; forces are in $\mu \mathrm{N}$.

\begin{tabular}{cccccc}
\hline Surface 1 & Surface 2 & Liquid & $\boldsymbol{F}_{\boldsymbol{a d h}}$ & $\boldsymbol{F}_{\boldsymbol{d}}$ & $\boldsymbol{F}_{\boldsymbol{a d h}} / \boldsymbol{F}_{\boldsymbol{d}}$ \\
\hline Glass & PDMS & Water & -1.96 & $1.66 \times 10^{-3}$ & $-1.18 \times 10^{3}$ \\
PMMA & PDMS & Water & -9.72 & $1.33 \times 10^{-4}$ & $-7.30 \times 10^{4}$ \\
Glass & PDMS & Ethanol & 1.49 & $1.90 \times 10^{-3}$ & $7.84 \times 10^{2}$ \\
PMMA & PDMS & Ethanol & 1.19 & $3.67 \times 10^{-4}$ & $3.24 \times 10^{3}$ \\
\hline
\end{tabular}




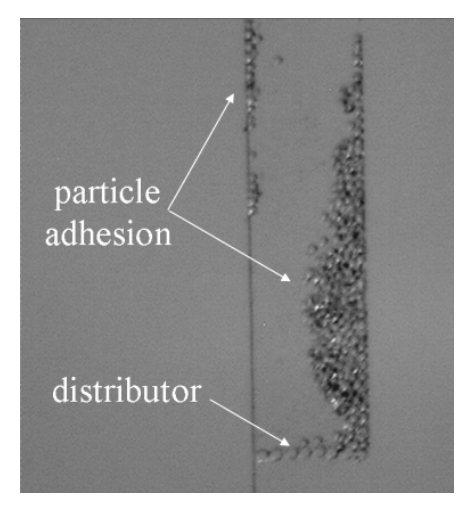

Fig. 3: Optical micrograph showing adhesion of glass particles to PDMS walls of the microchannel when deionized water is used as the fluidizing medium. The distributor was the circular pillars structure shown in Fig. 1(b).

Whilst the ratios of the adhesion to drag forces for the cases where ethanol is used as the fluidizing medium are similar in magnitude as when water is used (around 3 orders bigger), it is repulsive. Thus, Table 2 suggest that both the glass and PMMA microparticles should in practice not adhere to PDMS walls when ethanol is used as the fluidizing medium. Once again, our previous experiments (Zivkovic et al., 2013a) confirmed this proposition for glass particles, while new experiments confirmed this also to be the case for the PMMA microparticles; in both cases fluidization was smooth, stable and homogenous as shown in Fig. 4a \& 4b. These figures show that there was a sharp flat interface between the top of the bed and freeboard, typical of liquid fluidized beds. 


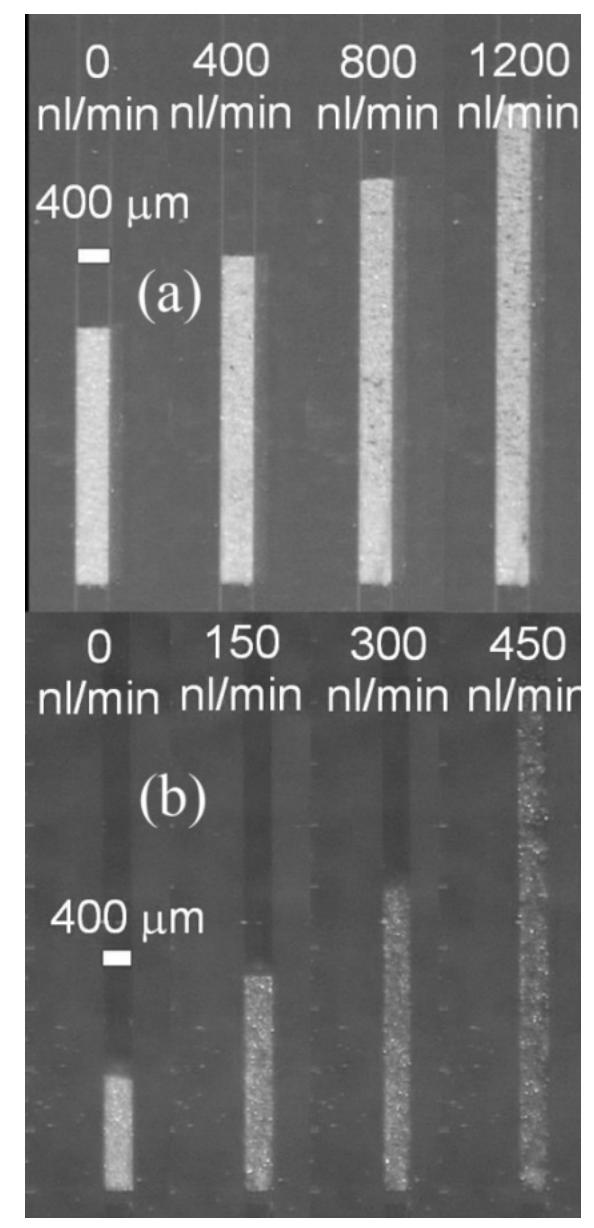

Fig. 4: Optical micrographs of successful fluidization with: (a) glass; and (b) PMMA microparticles in a PDMS microchannel at different ethanol flow rates. The distributors used for glass and PMMA particles fluidization were circular and rectangular pillars structures respectively (Fig. 1(b) and (c) respectively).

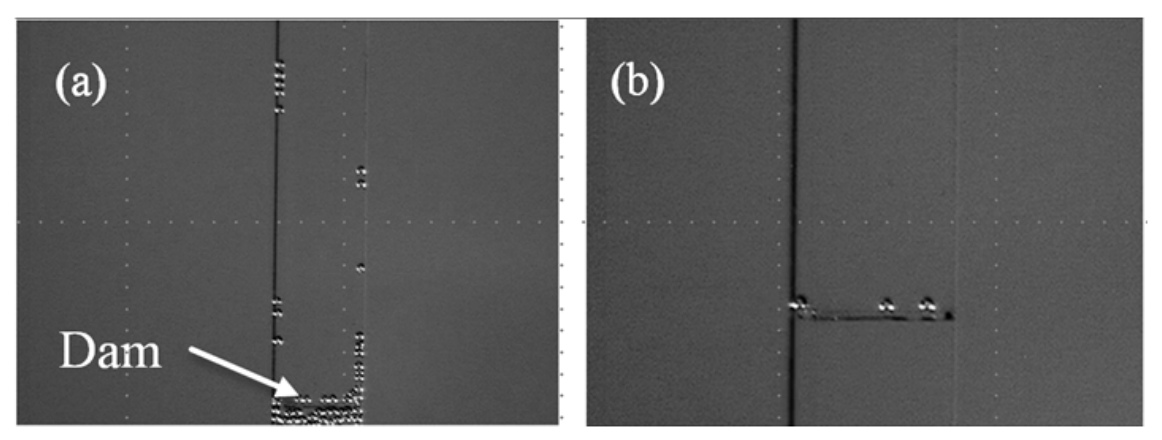

Fig. 5: Optical micrograph showing glass particles inside PDMS channels for Tween-80 aqueous solution as the fluidizing medium: (a) before elutriation; and (b) after elutriation. A dam distributor (Fig. 1(a)) was used here. 
Obviously, water as a fluidizing medium would be preferred in many applications. Therefore, we used an aqueous solution of non-ionic surfactant (Tween-80) as a fluidizing medium (Shukla and Henthorn, 2009) in an attempt to resolve the adhesion problem encountered when using pure water. Adhesion was still, however, observed, albeit to a lesser extent, with the microparticles only being observed to flow at superficial velocities several times the particle settling velocity, leading to their rapid elutriation from the bed, as can be seen in Fig. 5 (Zivkovic et al., 2013a).

We attempted to better understand our Tween-80 related observations using the acidbase approach of van Oss, Chaudhury and Good (van Oss, 2008) in conjunction with the surface tension components of Da Silva et al. (2011) for the hydrophobic tail and hydrophilic head of the Tween 80 molecule, which are summarised in Table 4. This analysis, which yields the interfacial energy estimates in Table 5, suggest that, when interacting with the glass particles, the Tween 80 molecules would prefer to attach via their tail. The modest magnitude of the free energy of this interaction $\left(6.2 \mathrm{~mJ} / \mathrm{m}^{2}\right)$ compared to that between the Tween molecule and PDMS (23.1 and $55.4 \mathrm{~mJ} / \mathrm{m}^{2}$ respectively) suggests, however, that the walls of the bed will be more fully covered by surfactant compared to the glass particles. The negative free energies associated with the interaction between either end of the Tween molecule and PDMS indicates both ends absorb to the bed walls, although the relative magnitudes of these energies suggests attachment by the tail group is more prevalent. Taken together, as illustrated in Fig. 6(a), the presence of Tween 80 molecules should prevent glass particle adhesion to the PDMS walls. This is, of course, in contrast to the experiments. We can hypothesize two possible explanations for this, neither being mutually exclusive, although they may occur to varying levels. The first is the possibility that the Tween film on both surfaces 
is incomplete, leading to the scenario illustrated in Fig. 6(b). The second possibility is adhesion between the head groups exposed on the glass particles and patches of the minority of Tween molecules adsorbed to the PDMS via their tail groups as illustrated in Fig. 6(c). Unfortunately, only a few Tween solution experiments could be performed before the micro-fluidized bed failed due to de-lamination and thus testing of these hypotheses was not possible as part of the work reported here; future work will, however, be undertaken to investigate this further by considering, for example, different concentration of the Tween surfactant as well as other surfactant molecules.

Table 4: Surface tension components (in $\mathrm{mJ} / \mathrm{m}^{2}$ ) of Tween 80 molecules (Da Silva et al. 2011)

\begin{tabular}{cccccc}
\hline Surfactant molecule end & $\gamma$ & $\gamma^{L W}$ & $\gamma^{A B}$ & $\gamma^{+}$ & $\gamma^{-}$ \\
\hline Hydrophilic moiety (head) & 48.19 & 45.61 & 2.58 & 0.04 & 41.52 \\
Hydrophobic moiety (tail) & 34.1 & 34.1 & 0 & 0 & 7 \\
\hline
\end{tabular}

Table 5: Calculated interfacial energy between solid surfaces, $i$, and Tween 80 molecules, $s$, immersed in water.

\begin{tabular}{cccc}
\hline \multirow{2}{*}{ Solid surface, $\boldsymbol{i}$} & \multicolumn{2}{c}{$\boldsymbol{\Delta \boldsymbol { G } _ { \text { iws } } \text { for Tween } \mathbf { 8 0 } ( \mathbf { m J } / \mathbf { m } ^ { \mathbf { 2 } } )}$} & \multirow{2}{*}{ Interaction for head/tail } \\
\cline { 2 - 3 } & Head group & Tail group & \\
\hline PDMS & -23.1 & -55.4 & attractive/attractive \\
Glass & 14.8 & -6.2 & repulsive/attractive \\
\hline
\end{tabular}

(a)

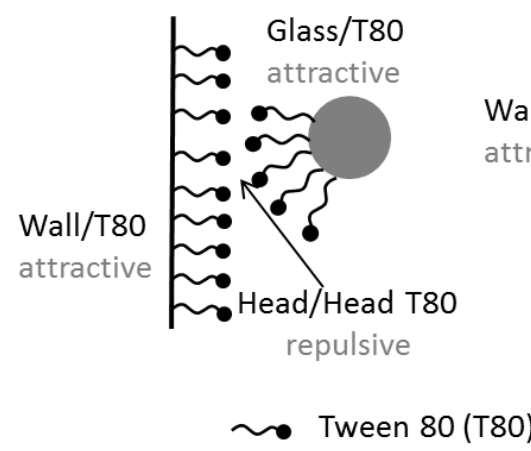

(b)

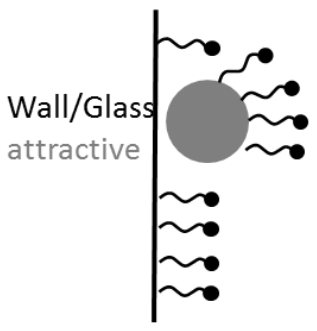

T80) (c)

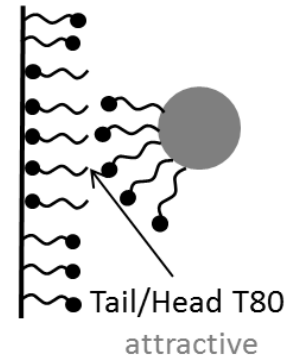

Glass particle

Fig. 6: Schematic representations of the Tween 80 molecule configurations in the PDMS/glass particle/aqueous Tween solution as suggested by the acid-base theory: (a) most common arrangement; (b) first hypothesised scenario to account for experimentally observed adhesion of particles; and (c) second hypothesised scenario to account for experimentally observed adhesion of particles. 


\section{Boundary Between Macro- and Micro-fluidization}

As stated in the Introduction to this paper, the boundary between macro- and microscale flows has long been set at a conduit cross-sectional size of around $1 \mathrm{~mm}$ (Gunther and Jensen, 2006; Hartman and Jensen, 2009). Here we use the approach outlined in the previous section to identify the boundary between the microfluidized bed and classical macro-scale fluidized bed for liquid fluidization. Fig. 7(a) shows the ratio of estimated adhesion forces to drag force as a function of particle diameter for the glass and PMMA particles inside a PDMS bed with water as the fluidizing medium; the -2 gradient of the lines in this figure arises from the adhesion force scaling directly with particle diameter, Eq. 3, whilst the drag force scales with the cube of the diameter, Eq. 6 . We can see from this figure that the adhesion force becomes comparable to the drag force (ratio below 10) for approximately $300 \mu$ m glass particles and 2.5 mm PMMA particles, while they are equal for approximately $1 \mathrm{~mm}$ and $8 \mathrm{~mm}$ glass and PMMA particle diameter respectively. Based on this analysis and assuming a bed-to-particle ratio of 10 , which is typical in micro-fluidized bed studies (Doroodchi et al., 2012; Potic et al., 2005; Zivkovic et al., 2013b), the rough boundary between micro-and macro-fluidization can be estimated to be between 1-10 mm for glass particles and between $10-80 \mathrm{~mm}$ for PMMA particles.

In most systems the adhesion forces are unlikely to be much greater than those associated with the PDMS/PMMA/water system, and so a cross-sectional size of the order of a cm is probably not an unreasonable upper limit for the boundary between micro- and macro-scale fluidization. In the case of the PDMS/glass/water system, the boundary drops to around $1 \mathrm{~mm}$, in line with commonly asserted boundary between micro- and macroscopic flow (Gunther and Jensen, 2006; Hartman and Jensen, 2009). 

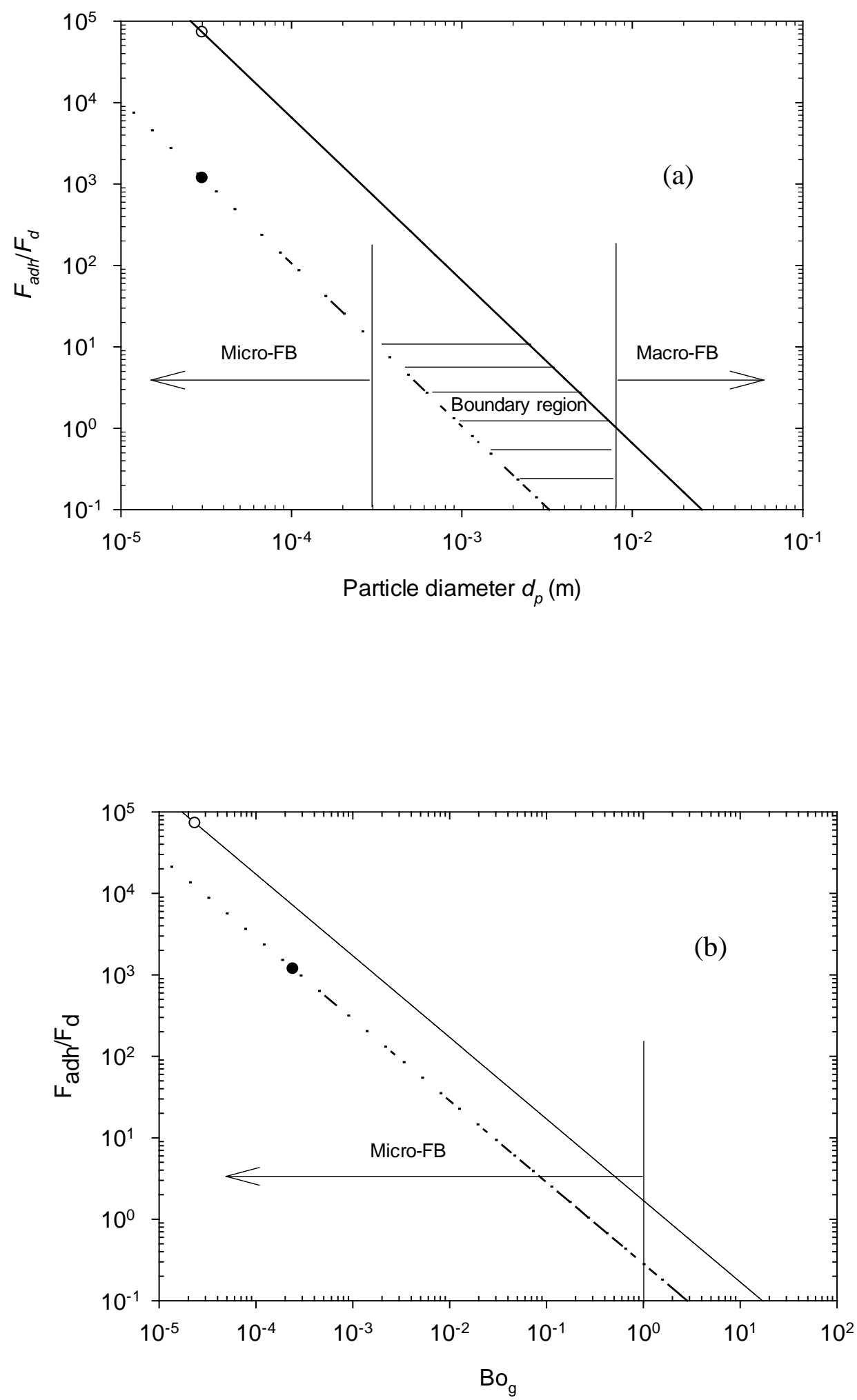

Fig. 7: Ratio of adhesion forces, $F_{a d h}$, to drag force, $F_{d}$, as a function of (a) particle diameter and (b) granular Bond number, $\mathrm{Bo}_{g}$, for glass (dashed line) and PMMA particles (solid line) with water as fluidizing medium in PDMS micro- fluidized bed. 
Solid and empty circles are points for glass and PMMA particles at the experimental condition in our study. The transition region where the adhesion and drag forces are comparable is shown as a hatched area in (a); this demarks the boundary between micro- and macro-fluidized beds.

Another way to quantify the importance of surface forces to body forces is the nondimensional granular Bond number

$\mathrm{Bo}_{\mathrm{g}}=\frac{\left(\rho_{p}-\rho_{f}\right) g d_{p}^{2}}{\gamma}$

where we used the mean value of surface tension of the particles for $\gamma$. Figure 7(b), which shows the ratio of the adhesion forces to body forces as a function of the granular Bond number, indicates that surface forces begin to dominate over gravity and related hydrodynamic drag for $\mathrm{Bo}_{\mathrm{g}}$ of approximately unity, and that micro-fluidization could be classified by the condition $\mathrm{Bog}_{\mathrm{g}}<<1$ as in our microfluidic fluidized beds. This is consistent with the above analysis.

\section{CONCLUSIONS}

Our experiments in microfluidized beds reveal a major difference from their classical macro-scale counterparts: the critical importance of surface forces, which have to be taken into account when designing micro-fluidized beds as adhesion of particles to the walls can prevent fluidization. We show here that the acid-base model of van Oss, Chaudhury and Good combined with the Derjaguin approximation (van Oss, 2008) can successfully predict the experimentally observed behaviour of glass and PMMA microparticles within a PDMS-based microfluidic beds using common liquid fluidizing media. We also use this theory to identify the granular Bond number that defines the boundary between micro- and macro-scale fluidization - based on a bed to particle size ratio of 10 , an upper limit of the micro-scale flow is likely to correspond to conduit 
cross-sectional sizes of around $1 \mathrm{~cm}$. This analysis also shows that cross-sectional sizes of $1 \mathrm{~mm}$, which is the commonly asserted boundary, is appropriate when working with glass particles fluidized by water within a PDMS conduit.

\section{ACKNOWLEDGEMENTS}

VZ acknowledge the University of Adelaide for his fellowship for research in microfluidics area. The microchannel fabrication was performed at the South Australian node of the Australian National Fabrication Facility under the National Collaborative Research Infrastructure Strategy.

\section{REFERENCES}

Abgrall, P., Gué, A.-M., 2007. Lab-on-chip technologies: making a microfluidic network and coupling it into a complete microsystems: a review. Journal of Micromechanics and Microengineering 17, R15.

Becker, H., Gärtner, C., 2008. Polymer microfabrication technologies for microfluidic systems. Analytical and Bioanalytical Chemistry 390, 89-111.

Chae, S.-K., Lee, C.-H., Lee, S.H., Kim, T.-S., Kang, J.Y., 2009. Oil droplet generation in PDMS microchannel using an amphiphilic continuous phase. Lab on a Chip 9, 19571961.

Charpentier, J.-C., 2005. Four main objectives for the future of chemical and process engineering mainly concerned by the science and technologies of new materials production. Chemical Engineering Journal 107, 3-17.

Clift, R., Seville, J.P.K., Moore, S.C., Chavarie, C., 1987. Comments on buoyancy in fluidized beds. Chemical Engineering Science 42, 191-194.

Clint, J.H., Wicks, A.C., 2001. Adhesion under water: surface energy considerations. International Journal of Adhesion and Adhesives 21, 267-273.

Da Silva, S.M., Filliben, J.J., Morrow, J.B., 2011. Parameters Affecting Spore Recovery from Wipes Used in Biological Surface Sampling. Applied and Environmental Microbiology 77, 2374-2380.

Della Volpe, C., Siboni, S., 2000. Troubleshooting of surface free energy acid-base theory applied to solid surfaces: The case of Good, van Oss and Chaudhury theory, in: Mittal, K.L., Andersson, H.R. (Eds.), Acid-base interactions: relevance to adhesion science and technology. VSP, Utrecht, The Netherlands, pp. 55-91.

Derksen, J.J., 2008. Scalar mixing by granular particles. AIChE Journal 54, 1741-1747.

Derksen, J.J., 2009. Scalar mixing with fixed and fluidized particles in micro-reactors. Chemical Engineering Research and Design 87, 550-556. 
Derveaux, S., Stubbe, B., Braeckmans, K., Roelant, C., Sato, K., Demeester, J., De Smedt, S., 2008. Synergism between particle-based multiplexing and microfluidics technologies may bring diagnostics closer to the patient. Analytical and Bioanalytical Chemistry 391, 2453-2467.

Di Felice, R., 1995. Hydrodynamics of liquid fluidisation. Chemical Engineering Science 50, 1213-1245.

Dittrich, P.S., Tachikawa, K., Manz, A., 2006. Micro Total Analysis Systems. Latest Advancements and Trends. Analytical Chemistry 78, 3887-3908.

Doroodchi, E., Peng, Z., Sathe, M., Abbasi-Shavazi, E., Evans, G.M., 2012. Fluidisation and packed bed behaviour in capillary tubes. Powder Technology 223, 131-136.

Dudukovic, M.P., 2009. Frontiers in Reactor Engineering. Science 325, 698-701.

Epstein, N., 2003. Liquid-solids fluidization, in: Yang, W.-c. (Ed.), Handbook of fluidization and fluid-particle systems. CRC Press, New York, USA, pp. 705-764.

Freitas, A.M., Sharma, M.M., 1999. Effect of surface hydrophobicity on the hydrodynamic detachment of particles from surfaces. Langmuir 15, 2466-2476.

Gunther, A., Jensen, K.F., 2006. Multiphase microfluidics: from flow characteristics to chemical and materials synthesis. Lab on a Chip 6, 1487-1503.

Haeberle, S., Zengerle, R., 2007. Microfluidic platforms for lab-on-a-chip applications. Lab on a Chip 7, 1094-1110.

Hartman, R.L., Jensen, K.F., 2009. Microchemical systems for continuous-flow synthesis. Lab on a Chip 9, 2495-2507.

Haswell, S.J., 2006. Chemical technology: All together now. Nature 441, 705-705.

Hu, Z., Kwon, G., Kim, C.-B., Kim, D., Lee, S.-H., 2010. Integration of movable structures in PDMS microfluidic channels. BioChip Journal 4, 117-122.

Jensen, K.F., 2001. Microreaction engineering -- is small better? Chemical Engineering Science 56, 293-303.

Lim, C.T., Zhang, Y., 2007. Bead-based microfluidic immunoassays: The next generation. Biosensors and Bioelectronics 22, 1197-1204.

Manz, A., Graber, N., Widmer, H.M., 1990. Miniaturized total chemical analysis systems: A novel concept for chemical sensing. Sensors and Actuators B 1, 244-248.

Martinelli, E., Suffredini, M., Galli, G., Glisenti, A., Pettitt, M.E., Callow, M.E., Callow, J.A., Williams, D., Lyall, G., 2011. Amphiphilic block copolymer/poly(dimethylsiloxane) (PDMS) blends and nanocomposites for improved fouling-release. Biofouling 27, 529-541.

Melin, J., Quake, S.R., 2007. Microfluidic Large-Scale Integration: The Evolution of Design Rules for Biological Automation. Annual Review of Biophysics and Biomolecular Structure 36, 213-231.

Potic, B., Kersten, S.R.A., Ye, M., van der Hoef, M.A., Kuipers, J.A.M., van Swaaij, W.P.M., 2005. Fluidization with hot compressed water in micro-reactors. Chemical Engineering Science 60, 5982-5990. 
Shukla, N., Henthorn, K., 2009. Effect of relative particle size on large particle detachment from a microchannel. Microfluidics and Nanofluidics 6, 521-527.

Squires, T.M., Quake, S.R., 2005. Microfluidics: Fluid physics at the nanoliter scale. Reviews of Modern Physics 77, 977.

Stone, H.A., Stroock, A.D., Ajdari, A., 2004. Engineering flows in small devices: Microfluidics towards lab-on-a-chip. Annual Review of Fluid Mechanics 36, 381-411.

van Oss, C.J., 1989. On the mechanism of the cold ethanol precipitation method of plasma protein fractionation. Journal of Protein Chemistry 8, 661-668.

van Oss, C.J., 2008. Chapter Two The Apolar and Polar Properties of Liquid Water and Other Condensed-Phase Materials, in: Carel, J.v.O. (Ed.), Interface Science and Technology. Elsevier, pp. 13-30.

West, J., Becker, M., Tombrink, S., Manz, A., 2008. Micro Total Analysis Systems: Latest Achievements. Analytical Chemistry 80, 4403-4419.

Whitesides, G.M., 2006. The origins and the future of microfluidics. Nature 442, 368373.

Whitesides, G.M., Ostuni, E., Takayama, S., Jiang, X., Ingber, D.E., 2001. Soft lithography in biology and biochemistry. Annual Review of Biomedical Engineering 3, 335-373.

Zdziennicka, A., 2010. The wettability of polytetrafluoroethylene and polymethylmethacrylate with regard to interface behaviour of Triton X-165 and short chain alcohol mixtures: I. Critical surface tension of wetting and adhesion work. Colloids and Surfaces A: Physicochemical and Engineering Aspects 367, 108-114.

Zivkovic, V., Biggs, M.J., Alwahabi, Z., 2010. Design of a distributor for a microfluidized bed, Chemeca Adelaide, Australia, p. 480.

Zivkovic, V., Biggs, M.J., Alwahabi, Z.T., 2013a. Experimental study of a liquid fluidization in a microfluidic channel. AIChE Journal 59, 361-364.

Zivkovic, V., Kashani, M.N., Biggs, M.J., 2013b. Experimental and theoretical study of a micro-fluidized bed. AIP Conference Proceedings 1542, 93-96.

Zivkovic, V., Zerna, P., Alwahabi, Z.T., Biggs, M.J., 2013c. A pressure drop correlation for low Reynolds number Newtonian flows through a rectangular orifice in a similarly shaped micro-channel. Chemical Engineering Research and Design 91, 1-6. 